\title{
ANZAHL DER BESTÄTIGTEN FÄLLE VON VIRUSHEPATITIS IN BRASILIEN ZWISCHEN 2010 UND 2015
}

\section{ORIGINALER ARTIKEL}

NUNES, Filipe Sales', FACCO, Lucas², FECURY, Amanda Alves³, ARAÚJO, Maria Helena Mendonça de ${ }^{4}$, OLIVEIRA, Euzébio de ${ }^{5}$, DENDASCK, Carla Viana ${ }^{6}$, SOUZA, Keulle Oliveira da ${ }^{7}$, DIAS, Claudio Alberto Gellis de Mattos $^{8}$

NUNES, Filipe Sales. Et al. Anzahl der bestätigten Fälle von Virushepatitis in Brasilien zwischen 2010 und 2015. Revista Científica Multidisciplinar Núcleo do Conhecimento. Jahrgang 05, Ed. 11, Vol. 25, S. 71-80. November 2020. ISSN: 24480959, Zugangslink:

https://www.nucleodoconhecimento.com.br/gesundheit/virushepatitis, DOI: 10.32749/nucleodoconhecimento.com.br/gesundheit/virushepatitis

\section{ZUSAMMENFASSUNG}

Virale Hepatitis ist Infektionskrankheiten, die die Leber angreifen, und ihre causatous Erreger sind Viren. Diese Studie soll die Anzahl der bestätigten Fälle von Virushepatitis in Brasilien zwischen 2010 und 2015 nachweisen. Eine Suche wurde in der DATASUS-Datenbank auf der Website (http://datasus.saude.gov.br/) durchgeführt. Hepatitis stellt in Brasilien ein großes Problem der öffentlichen Gesundheit dar. Von den Infizierten besteht ein großer Teil aus männlichen

\footnotetext{
${ }^{1}$ Computernetzwerktechniker, Absolvent des Bundesinstituts Amapá (IFAP).

${ }^{2}$ Student des Medizinkurses der Föderalen Universität Amapá (UNIFAP).

${ }^{3}$ Biomedizin, PhD in Tropenkrankheiten, Professor und Forscher des Medizinischen Studiengangs der Föderalen Universität Amapá (UNIFAP).

${ }^{4}$ Arzt, Professor und Forscher des Medizinischen Studiengangs der Föderalen Universität Amapá (UNIFAP).

${ }^{5}$ Biologe, PhD in Topische Krankheiten, Professor und Forscher des Leibeserziehungskurses der Föderalen Universität Pará (UFPA).

${ }^{6}$ Theologe, PhD in Psychoanalyse, Forscher am Zentrum für Forschung und Höhere Studien - CEPA.

${ }^{7}$ Soziologe, Master-Student in Anthropischen Studien im Amazonas, Mitglied der Forschungsgruppe "Labor für Bildung, Umwelt und Gesundheit" (LEMAS/UFPA).

${ }^{8}$ Biologe, PhD in Theorie- und Verhaltensforschung, Professor und Forscher des Graduiertenprogramms für berufliche und technologische Bildung (PROFEPT), Bundesinstitut für Amapá (IFAP).
}

RC: 67677

Verfügbar in: https://www.nucleodoconhecimento.com.br/gesundheit/virushepatitis 
Individuen, und die sichtbar geringere Nachfrage nach Gesundheitsdiensten ist ein wichtiger Faktor für diesen Befund. Hepatitis $B$ und $C$ sind die häufigsten bei Virushepatitis und einer der wichtigsten und beitragenden Faktoren für die Rate der infizierten Hepatitis-Viren ist ihre Koinfektion mit HIV. Labortests (Immunoassay, molekulare Tests) sollten durchgeführt werden, um Marker zu erkennen und das ätiologische Mittel zu bestimmen, das die Pathologie verursacht.

Schlagworte: Epidemiologie, Virus, Hepatitis.

\section{EINFÜHRUNG}

Virale Hepatitis ist Infektionskrankheiten, die die Leber angreifen. Sie gelten in Brasilien aufgrund ihrer großen Zahl bestätigter Fälle als Problem der öffentlichen Gesundheit (MARGREITER et al., 2015; LEITE, et al., 2020).

Die entdeckten ätiologischen Wirkstoffe der Virushepatitis sind die Viren HAV, HBV, $\mathrm{HCV}$, VHD und VHE, die gemeinsam die einfache Bindung mit Leberzellen haben. Sie zeigen Unterschiede in ihrer klinischen Form und epidemiologischen Merkmalen (NUNES et al., 2016).

Hepatitis $A(\mathrm{VHA})$ und $E(\mathrm{VHE})$ haben ihre Viren über den fäkal-oralen Weg übertragen und können in kontaminiertem Wasser und Lebensmitteln gefunden werden. Der Mangel an angemessener Behandlung des verbrauchten Wassers und der unzureichende Umgang mit Lebensmitteln sind die Hauptfaktoren für die Ausbreitung des Virus. Die Krankheit, wenn Symptome präsentieren, kann eine Abnahme des Appetits, Fieber und Veränderungen in der Urinfarbe des infizierten Individuums verursachen (MOCBEL et al., 2016).

Das Hepatitis-A-Virus hat einen wirksamen Impfstoff, der als sicher gilt und eine Immunität von mehreren von 5 bis 10 Jahren aufrechterhalten kann. Der Impfstoff ist in zwei Dosen unterteilt und kann auf Kinder ab dem ersten Lebensjahr angewendet werden (FERREIRA et al., 2014). Hepatitis E hat einen Impfstoff, aber nicht auf

RC: 67677

Verfügbar in: https://www.nucleodoconhecimento.com.br/gesundheit/virushepatitis 
globaler Ebene. Ursprünglich 2012 auf den Markt gebracht und in China produziert, gilt es bereits als wirksam (NUNES et al., 2016).

Hepatitis-C-Virus (HCV) wird in erster Linie durch Bluttransfusion übertragen, und auch durch Geschlechtsverkehr, angeborene Form, und teilen von scharfen Gegenständen oder persönliche Hygiene. Hepatitis C hat eine kompliziertere Diagnose aufgrund der Komplexität seines Virus. In weniger als der Hälfte der Fälle ist es nicht möglich, die mechanische Quelle der Infektion zu identifizieren. Die meisten Fälle von Hepatitis C erreichen die chronische Form, ohne Symptome zu präsentieren. Sie manifestieren sich in der Regel erst nach Jahren der Infektion, wenn Individuen sich in einem schwereren Stadium befinden. Die Minderheit der Fälle geht in der Regel zu Leberzirrhose oder Leberkrebs (GUSMÃO et al., 2017).

Die Behandlung variiert je nach und Genotyp des Virus, mit Medikamenten, um die Reproduktion des Virus zu verhindern, wodurch die Verschlechterung der Infektion reduziert wird. Die Dauer der Behandlung kann von 48 bis 72 Wochen betragen, wo es zu einer enormen Abnahme der Viruslast beim Patienten, aber nicht zu einem vollständigen Aussterben des Virus (SILVA et al., 2014). Ein Hepatitis-C-VirusImpfstoff wurde noch nicht entwickelt. Eine der Möglichkeiten, die Ausbreitung des Virus zu verhindern, ist die Mobilisierung von Risikogruppen, wie Drogenkonsumenten und Gesundheitsexperten (GUSMÃO et al., 2017).

Hepatitis B (HBV) hat sein Virus vertikal übertragen, von der Mutter auf das Kind zum Zeitpunkt der Entbindung oder durch Stillen übertragen. Auch durch den Austausch scharfer Gegenstände wie Nadeln und Zange, Bluttransfusion und sexuelle Form (FRANCISCO et al., 2015).

Hepatitis B und D können in zwei klinischen Formen auftreten: akut und chronisch. Wenn sie Symptome zeigen, sind sie identisch, wie körperliches Unwohlsein; Veränderungen in der Urinfärbung; Kot; Gelbfärbung der Haut und der Augen (BRASIL, 2014; BRASIL, 2014a).

RC: 67677

Verfügbar in: https://www.nucleodoconhecimento.com.br/gesundheit/virushepatitis 
Das Hepatitis-D-Virus (VHD) erfordert direkt, dass das Hepatitis-B-Virus (HBV) infektiös ist, so dass die Übertragungswege identisch sind, da es für eine Person nicht möglich ist, sich ohne HBV mit HBV zu infizieren. Hepatitis-B-Virus-Impfstoff ist auch wirksam für die Immunisierung gegen Hepatitis D aufgrund der Beziehung der Gelenkvirus-Infektion (NUNES et al., 2016).

\section{TOR}

Zeigen Sie die Anzahl der bestätigten Fälle von Virushepatitis in Brasilien zwischen 2010 und 2015.

\section{SYSTEM}

Eine Suche wurde in der DATASUS-Datenbank auf der Website (http://datasus.saude.gov.br/) durchgeführt, aus der die Daten entfernt wurden, und folgt den Schritten: Im Menü der Website wählten wir den Reiter "Zugriff auf Informationen", dann die Option "TABNET-Gesundheitsinformationen", dann die Option "Epidemiologien und Morbidität". Nach dem Laden der Seite wurde die Gruppe "Krankheiten und Krankheiten der Meldung- Ab 2007 (SINAN)" ausgewählt und die Option "Hepatitis" ausgewählt und im Tab "Geografische Abdeckung" wurde die Option "Brasilien nach Region, UF und Gemeinde" ausgewählt. In der Spaltenoption haben Sie "Nicht aktiv", "Geschlecht", "Altersgruppe", "Klasse" ausgewählt. Ätiologische", "Source Mecan Infection", "Class. Finale", "Klinische Form", "Schooling". Für jeden in der Spalte ausgewählten Artikel wurden in der Zeile immer "Jahresdiag/Symptome" und im Zeitraum 2010 bis 2015 immer die Jahre 2010 bis 2015 verwendet. Die Daten wurden in der Excel-Anwendung, einer Komponente der Microsoft Corporation Office-Suite, kompiliert. Die bibliographische Forschung wurde in wissenschaftlichen Artikeln mit Computern aus dem Computerlabor des Bundesinstituts für Bildung, Wissenschaft und Technologie von Amapá, Macapá Campus, unter: Rodovia BR 210 KM 3, s/n - Bairro Brasil Novo durchgeführt. CEP: 68.909-398, Macapá, Amapá, Brasil.

RC: 67677

Verfügbar in: https://www.nucleodoconhecimento.com.br/gesundheit/virushepatitis 


\section{ERGEBNISSE}

Abbildung 1 zeigt die Anzahl der bestätigten Fälle von Virushepatitis in Brasilien zwischen 2010 und 2015. Zwischen 2010 und 2013 zeigen die Daten einen Anstieg der Fallzahlen. Zwischen 2013 und 2015 ist ein Rückgang der VirushepatitisInfektionen zu verzeichnen.

Abbildung 1 Anzahl der bestätigten Fälle von Virushepatitis in Brasilien zwischen 2010 und 2015.

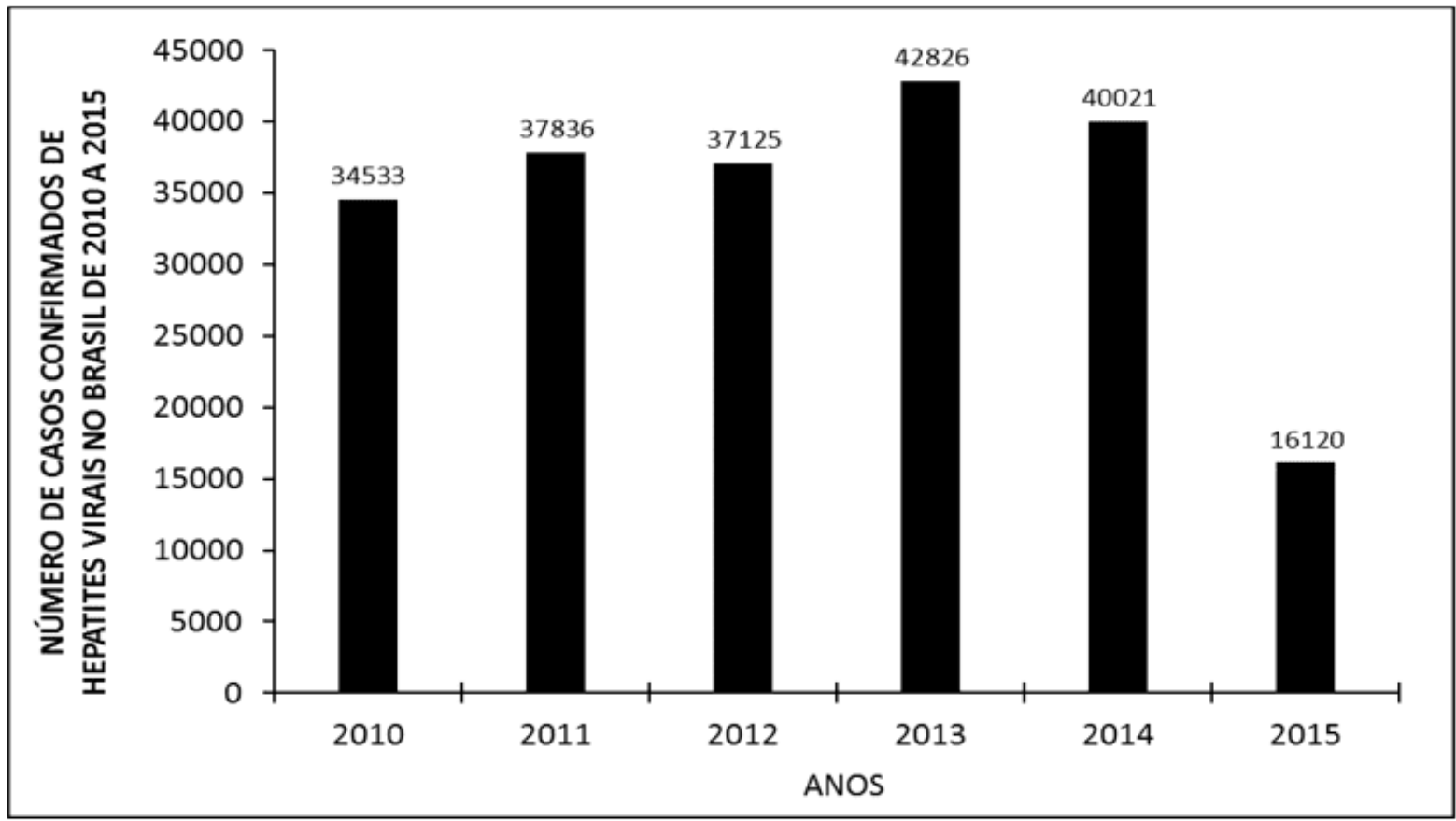

Abbildung 2 zeigt die Anzahl der bestätigten Fälle von Virushepatitis in Brasilien zwischen 2010 und 2015 nach Geschlecht. Die Daten zeigen eine höhere Anzahl von Fällen bei Männern im Vergleich zu Frauen.

$\mathrm{RC}: 67677$

Verfügbar in: https://www.nucleodoconhecimento.com.br/gesundheit/virushepatitis 
Abbildung 2 Anzahl der bestätigten Fälle von Virushepatitis in Brasilien zwischen 2010 und 2015 nach Geschlecht.

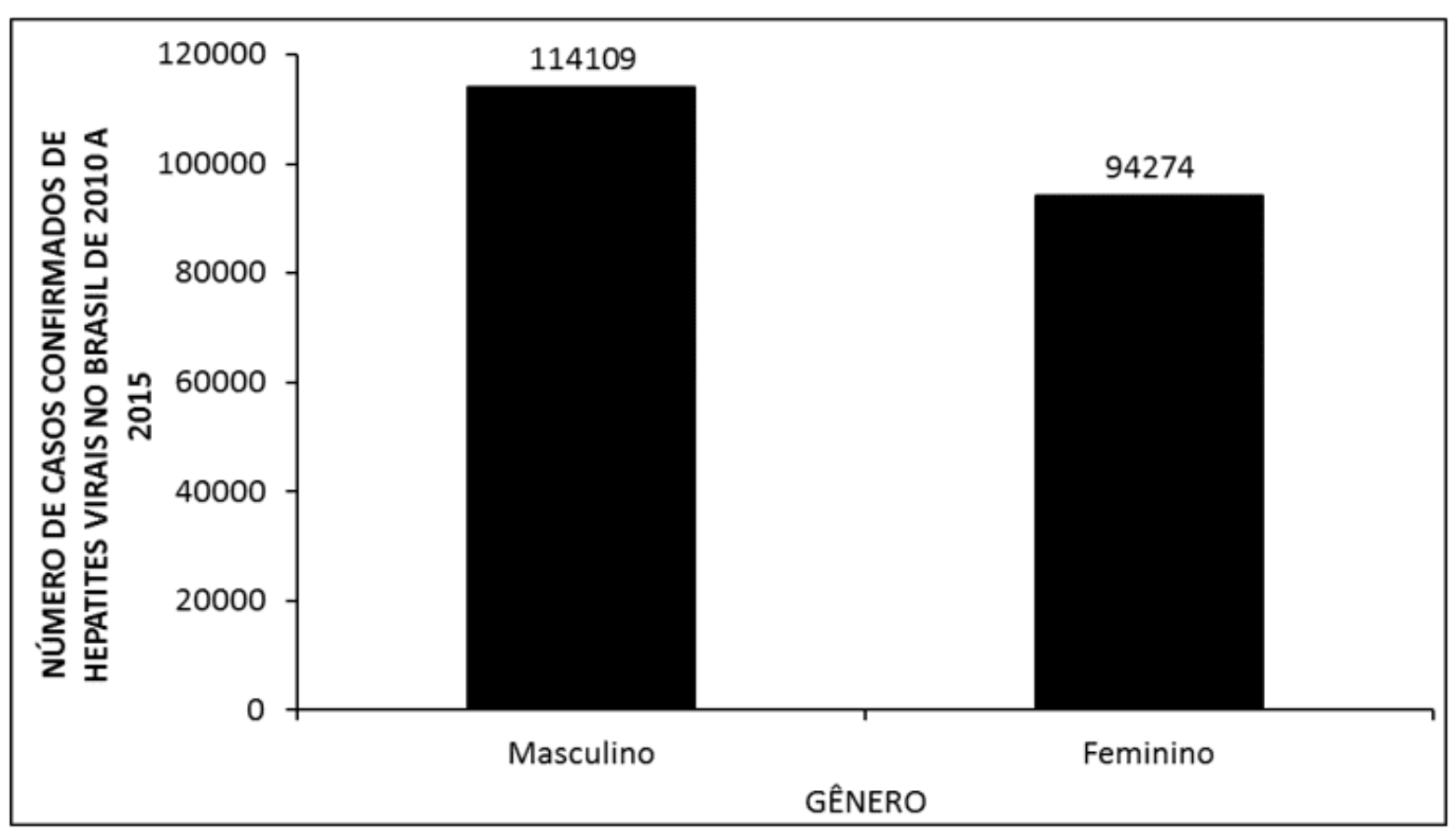

Abbildung 3 zeigt die Anzahl der bestätigten Fälle von Virushepatitis in Brasilien zwischen 2010 und 2015 nach Altersgruppen. Die meisten Fälle treten in der Altersgruppe zwischen 40 und 59 Jahren auf, die zweitgrößte Zahl zwischen 20 und 39 Jahren.

RC: 67677

Verfügbar in: https://www.nucleodoconhecimento.com.br/gesundheit/virushepatitis 
Abbildung 3 Anzahl bestätigter Fälle von Virushepatitis in Brasilien zwischen 2010 und 2015 nach Altersgruppe

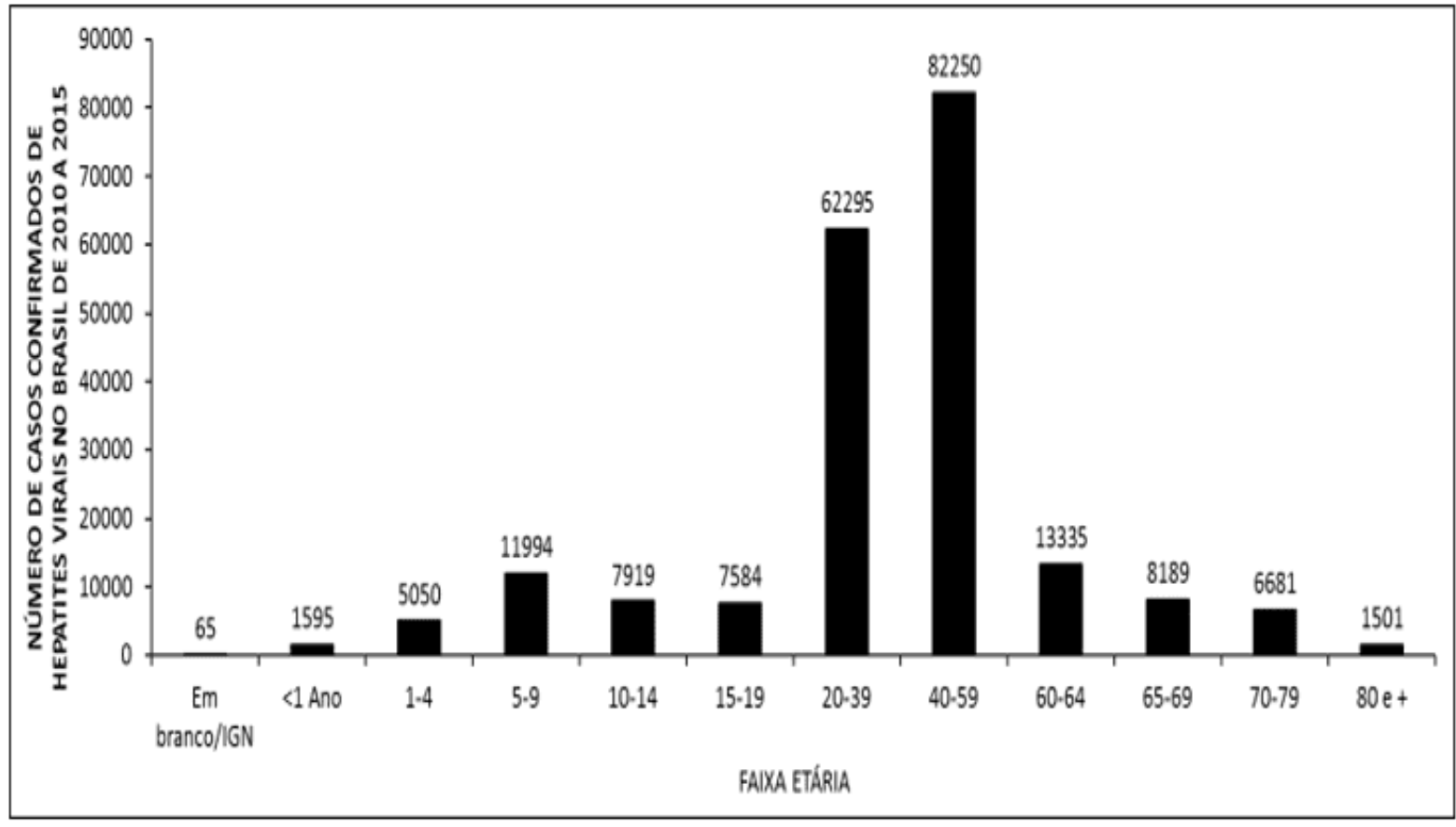

Abbildung 4 zeigt die Anzahl der bestätigten Fälle von Virushepatitis in Brasilien zwischen 2010 und 2015 nach ätiologischer Klasse, die höchste Rate der Krankheit, die mit der Darstellung des Virus C die zweitgrößte mit Virus B auftritt.

RC: 67677

Verfügbar in: https://www.nucleodoconhecimento.com.br/gesundheit/virushepatitis 
Abbildung 4 Anzahl der bestätigten Fälle von Virushepatitis in Brasilien von 2010 bis 2015 nach ätiologischer Klasse.

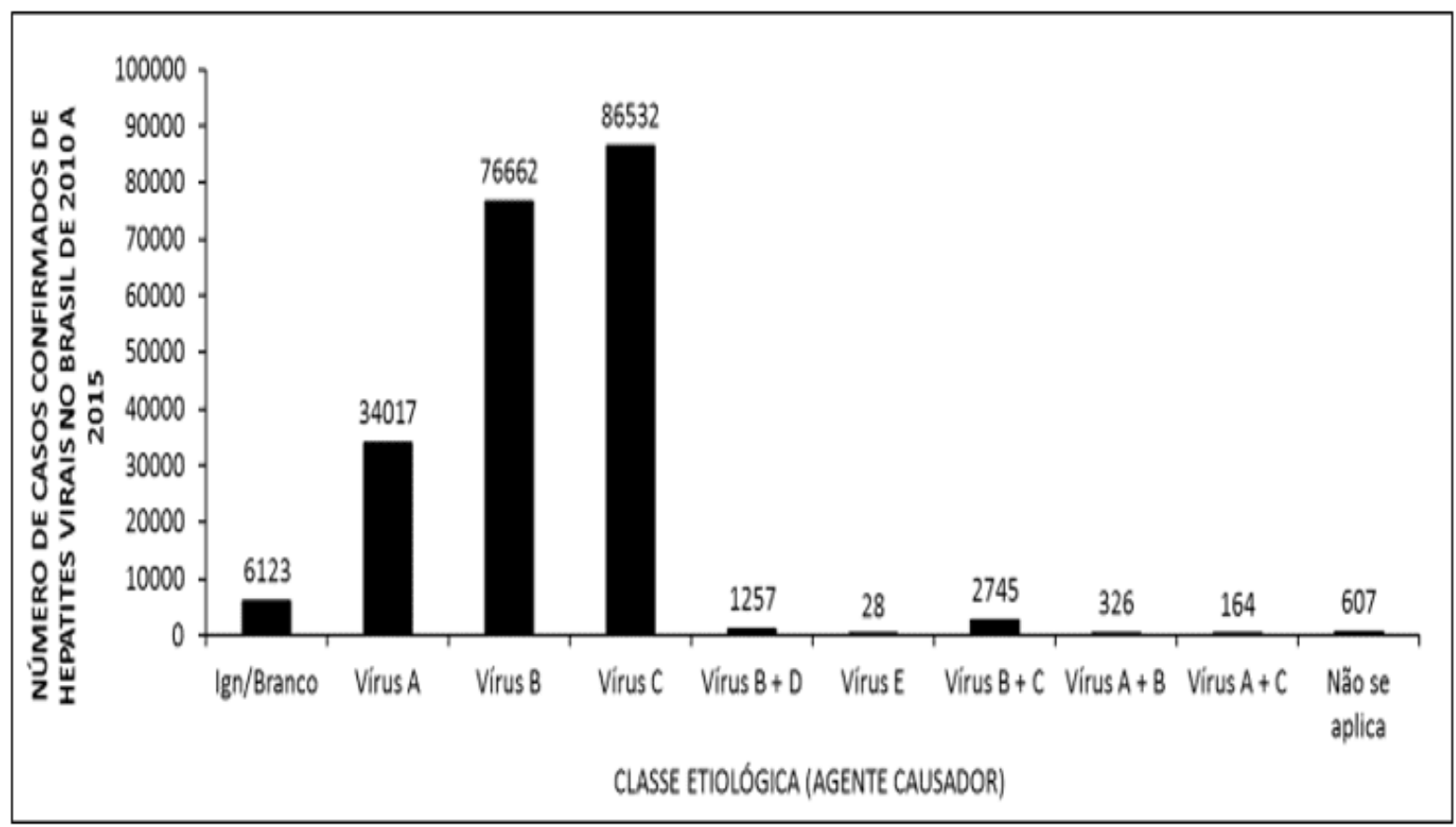

Abbildung 5 zeigt die Anzahl der bestätigten Fälle von Virushepatitis in Brasilien zwischen 2010 und 2015 durch mechanische Kontaminationsquelle, was zeigt, dass die größte Anzahl von Fällen, in denen die mechanische Quelle erkannt wird, durch sexuelle Übertragung und zweitens durch Lebensmittel/Wasser verursacht wurde.

RC: 67677

Verfügbar in: https://www.nucleodoconhecimento.com.br/gesundheit/virushepatitis 
Abbildung 5 Abbildung mit der Anzahl der bestätigten Fälle von Virushepatitis in Brasilien von 2010 bis 2015 nach mechanischer Quelle.

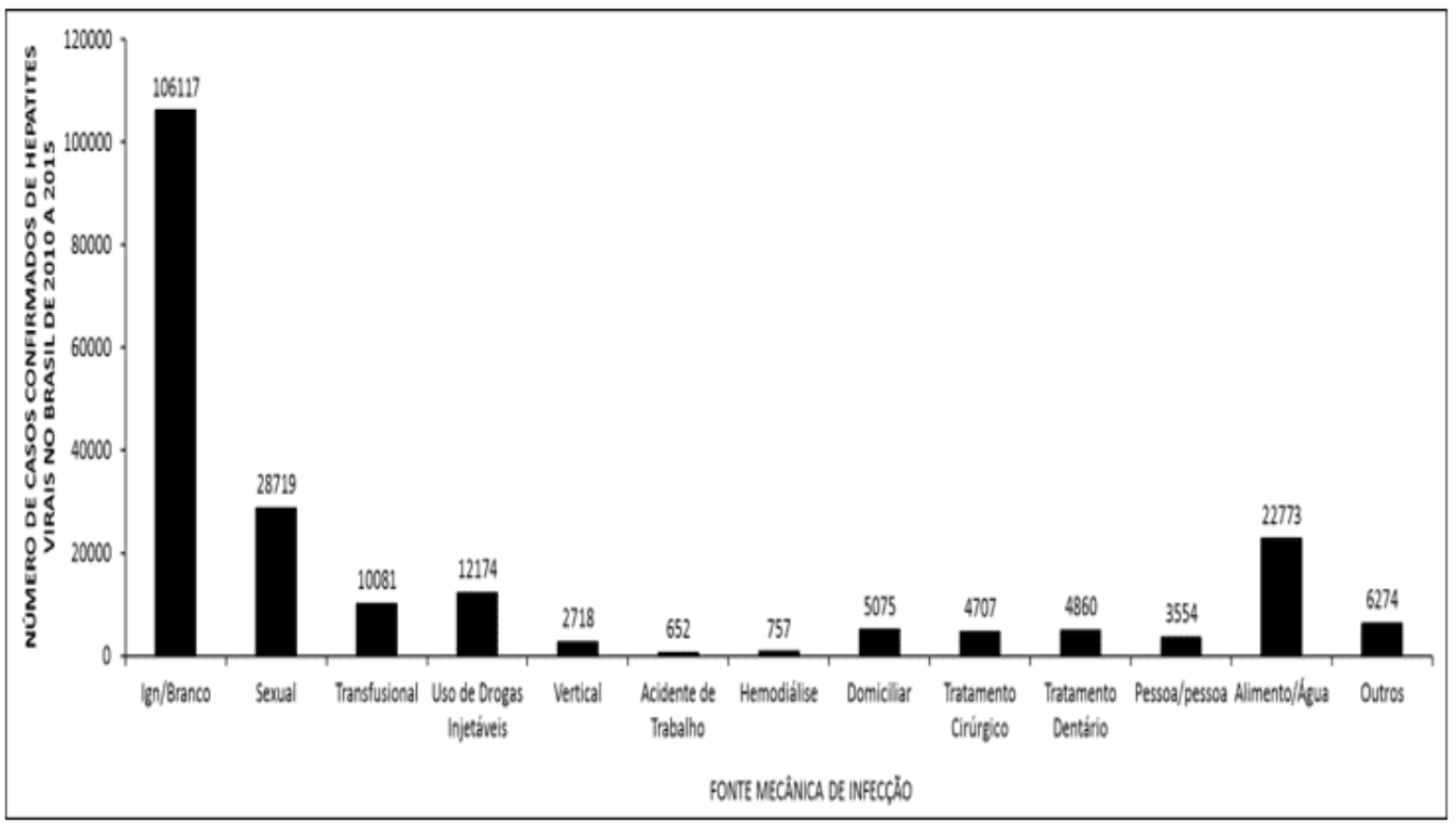

Abbildung 6 zeigt den prozentualen Anteil der bestätigten Fälle von Virushepatitis in Brasilien zwischen 2010 und 2015 nach seiner endgültigen Einstufung. Es wurde festgestellt, dass der höchste Prozentsatz der Diagnosen im Labor durchgeführt wurde.

RC: 67677

Verfügbar in: https://www.nucleodoconhecimento.com.br/gesundheit/virushepatitis 
Abbildung 6 Abbildung mit dem Prozentsatz der bestätigten Fälle von Virushepatitis in Brasilien nach endgültiger Einstufung zwischen 2010 und 2015.

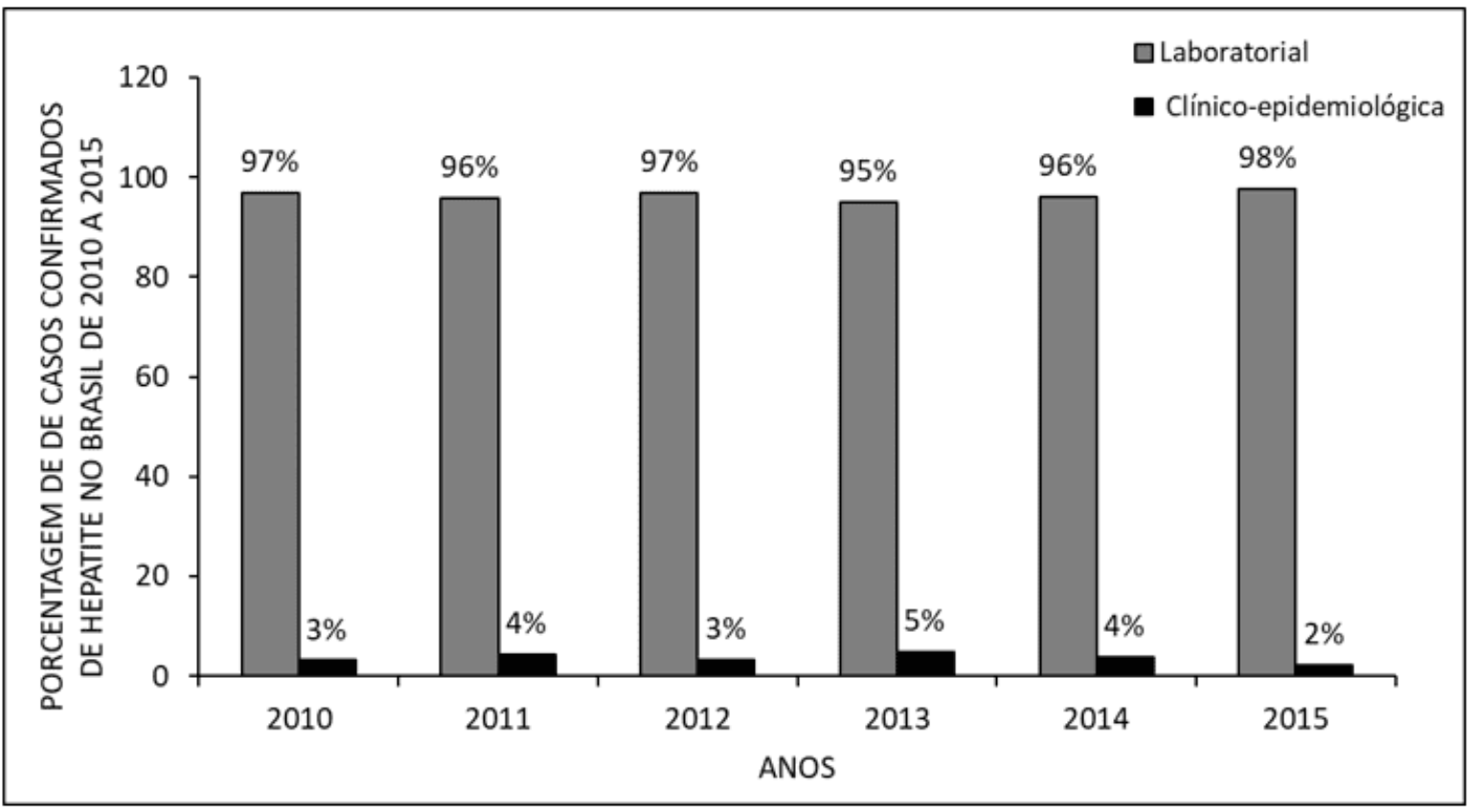

Abbildung 7 zeigt die Anzahl der bestätigten Fälle von Virushepatitis in Brasilien nach klinischer Form zwischen 2010 und 2015, was zeigt, dass die höchste Inzidenz in Form von chronischer Hepatitis/Träger ist.

RC: 67677

Verfügbar in: https://www.nucleodoconhecimento.com.br/gesundheit/virushepatitis 
Abbildung 7 Anzahl bestätigter Fälle von Virushepatitis in Brasilien von 2010 bis 2015 nach klinischer Form

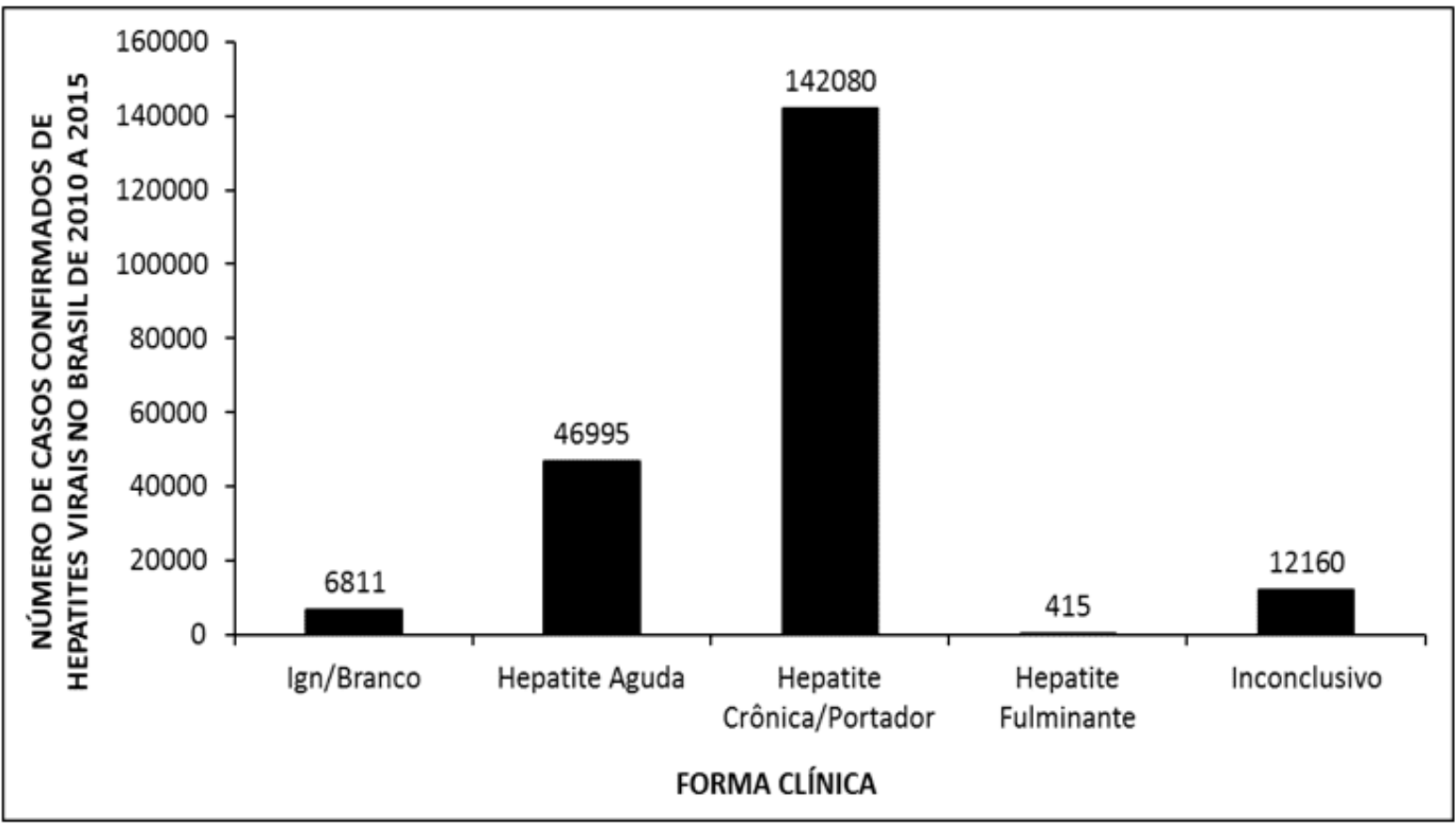

Abbildung 8 zeigt die Zahl der bestätigten Fälle von Virushepatitis in Brasilien zwischen 2010 und 2015 entsprechend dem Bildungsstand der Infizierten. Dies zeigt, dass die meisten Fälle, in denen die Person ihre Ausbildung identifiziert, bei Personen mit abgeschlossener Weiterführende Schule und an zweiter Stelle mit unvollständiger 5 . bis 8 . Klasse auftritt.

RC: 67677

Verfügbar in: https://www.nucleodoconhecimento.com.br/gesundheit/virushepatitis 
Abbildung 8 Anzahl der bestätigten Fälle von Virushepatitis in Brasilien zwischen 2010 und 2015 durch Die Ausbildung von Einzelpersonen.

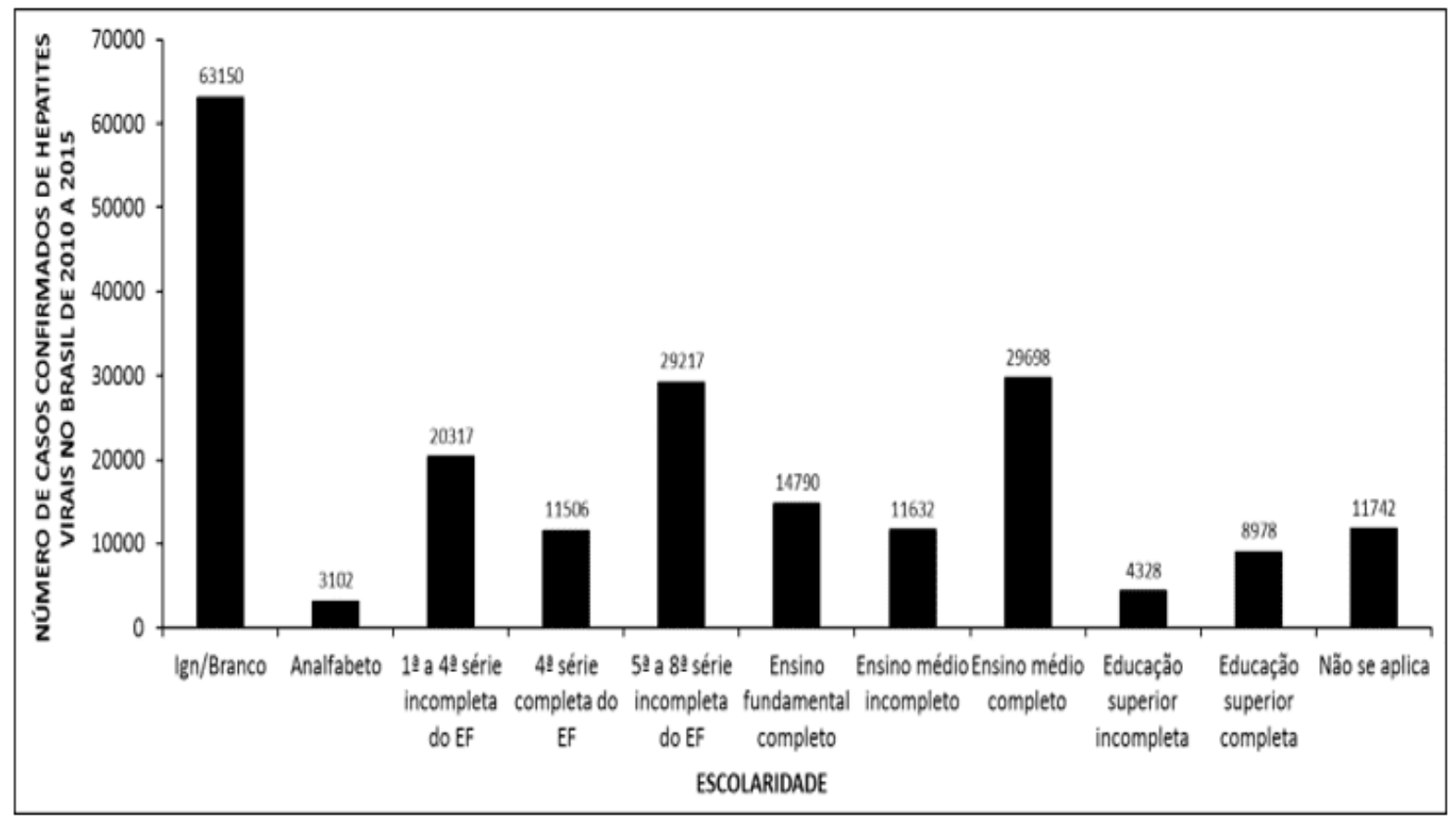

\section{DISKUSSION}

Virale Hepatitis ist ein umfassendes Problem der öffentlichen Gesundheit in Brasilien. Durch die Berücksichtigung mehrerer Faktoren, wie die Unregelmäßigkeit der Verteilung der Gesundheitsdienste und die damit einhergehende Ungleichheit der diagnostischen Technologie, zusätzlich zu den wirtschaftlichen und sozialen Ungleichheiten, ist es möglich, einige der Gründe zu verstehen, die mit der hohen Zahl von Hepatitis im Land zusammenhängen. Darüber hinaus tragen ungenaue Kenntnisse über die Anzahl der Infizierten und unzureichende Unterweisungen zu den ätiologischen Wirkstoffen der Virushepatitis und deren Übertragungsmittel auch negativ zur Dynamik des Fortschritts der Pathologie in Brasilien bei (FERREIRA und SILVEIRA, 2004).

Unter den Personen, die mit den verschiedenen Hepatitis-verursachenden Viren infiziert sind, ist die Mehrheit männlich. Zahlreiche Studien deuten darauf hin, dass

$\mathrm{RC}: 67677$

Verfügbar in: https://www.nucleodoconhecimento.com.br/gesundheit/virushepatitis 
Männer tendenziell weniger Nachfrage nach medizinischen Dienstleistungen im Allgemeinen ausüben und in Anbetracht dieser Tatsache mehr Todesfälle aufgrund chronischer Krankheiten erleiden (GOMES et al., 2007). Zu den Hauptgruppen, die für eine Hepatitis-Virus-Infektion gefährdet sind, insbesondere HBV, gibt es: Gesundheitsexperten, Menschen, die intravenöse Medikamente verwenden, Hämodialyse-Patienten, Sex-Profis und männliche homosexuelle Personen (FERREIRA und SILVEIRA, 2004).

Durch Daten aus dem Epidemiologischen Bulletin 2015 zur Viralen Hepatitis ist es möglich, die wichtigsten Altersgruppen zu überprüfen, in denen Hepatitis-Fälle vorherrschen. Im Falle von Hepatitis A tritt die Infektion meist bei Kindern unter 10 Jahren auf, und der Höhepunkt liegt im Bereich zwischen 5 und 6 Jahren. In Bezug auf Hepatitis B tritt der Höhepunkt der Fälle im Altersbereich von 20 und 30 Jahren auf. Bei Hepatitis $C$ wird festgestellt, dass die höchste Fallrate bei Personen im Alter von 40 bis 60 Jahren auftritt (BRASIL, 2015). Eine wichtige Beobachtung ist der signifikante Anstieg - 97,7\% - der Hepatitis-A-Übertragung bei Männern zwischen 20 und 39 Jahren durch sexuelle Mittel, laut dem Epidemiologischen Bulletin of Viral Hepatitis 2018 (BRASIL, 2018a). Der sexuelle Weg der Übertragung von Hepatitis C ist Gegenstand von Debatten, da HCV im Gegensatz zum anerkannten Potenzial für die sexuelle Übertragbarkeit von Hepatitis B eine sexuelle Infektiosität aufweist, die in begrenzter Weise auftritt und daher nicht als STDs (Sexuell-übertragbare Krankheit) eingestuft wird (ALMEIDA und MARTINS, 2015).

In Brasilien gab es zwischen 1999 und 2018632.814 bestätigte Fälle von Virushepatitis. Von diesen Fällen beziehen sich 36,8\% (233.027) auf Hepatitis B, 36,1\% (228.695) auf Hepatitis C, die die höchsten Zahlen darstellen. Hepatitis A macht 26,4 \% der Gesamtzahl (167.108) aus, zusätzlich zu 0,7 \% Hepatitis D (3.984), wobei letztere Hepatitis mit der niedrigsten Anzahl von Meldungen und einem umfassenden Unterschied in der Zahl der anderen ist. Hepatitis $C$ hat seit 2015 die Hepatitis-B-Zahlen bei den Inzidenzraten jährlich übertroffen (BRASIL,2019). Ein zu berücksichtigender Punkt ist die Koinfektion mit dem 
humanen Immundefizienzvirus (HIV) und Hepatitis B (HBV) und C (HCV), da 70 bis 90 \% der HIV-infizierten Patienten Marker einer bestandenen HBV-Infektion haben (FARIAS et al., 2012)

Die klinische Diagnose der virushepatitis tritt durch Anzeichen und Symptome des Patienten auf, und Personen, die das Virus haben, können akute, chronische und sogar asymptomatische symptomatische Bedingungen aufweisen. Bei akuter Hepatitis kann der Patient mit Unwohlsein, Müdigkeit, Übelkeit, Cholurie (verdunkelter Urin), Magersucht, Gelbsucht und weißlich gefärbten Stühlen auftreten. Im Falle eines Bildes von chronischer Hepatitis können manchmal Personen asymptomatisch sein, jedoch können Leberbeteiligungen nach einer bestimmten Zeit auftreten, wie Leberzirrhose, Leberfibrose und sogar hepatozelluläres Karzinom (je nach Evolution können andere Organe beeinträchtigt werden). Die Diagnose erfolgt meist in der chronischen Phase der Krankheit (hauptsächlich bei Hepatitis B und C). Auf Laborebene ist es möglich, Marker für Hepatitis zu erkennen, und die für diesen Prozess verwendeten Inhalte sind orale Flüssigkeiten, Blut, Serum oder Plasma des infizierten Patienten, durch Techniken, die als Immunoassays bezeichnet werden. Solche Verfahren zielen darauf ab, das Antigen selbst oder Antikörper zu erkennen, und es gibt verschiedene Arten: immunenzymatische Assays, Lumineszenz- und Schnelltests. Darüber hinaus gibt es auch molekulare Tests, basierend auf Polymerase-Kettenreaktion (PCR) (BRASIL, 2018b und SBI, 2003)

Die Schulbildung ist einer der Faktoren, die epidemiologisch berücksichtigt werden, um die Auswirkungen von Hepatitis in Brasilien zu verstehen. In Bezug auf bestätigte Fälle von Virushepatitis wird beobachtet, dass ein großer Teil der Menschen mit Schulbildung von der fünften bis achten Klasse der Grundschule und von 1999 bis 2018 bei allen bestätigten Fällen 21.844 personen, die diesem Profil entsprechen (niedriger nur in der Kategorie "ignoriert", durch Daten aus dem Epidemiologischen Bulletin der Viralen Hepatitis 2019) (BRASIL, 2019).

$\mathrm{RC}: 67677$

Verfügbar in: https://www.nucleodoconhecimento.com.br/gesundheit/virushepatitis 


\section{SCHLUSSFOLGERUNGEN}

Hepatitis stellt in Brasilien ein großes Problem der öffentlichen Gesundheit dar, und Faktoren wie die ungleiche Verteilung der Gesundheitsdienste, sozioökonomische Disparitäten und andere Probleme tragen zu diesem Durcheinander bei.

Von den Infizierten besteht ein großer Teil aus männlichen Individuen, und die sichtbar geringere Nachfrage nach Gesundheitsdiensten ist ein wichtiger Faktor für diesen Befund. Aus diesem Grund ist es, Sie neigen dazu, mehr chronische Beteiligung aufgrund der Verzögerung bei der Suche nach einem Arzt leiden. Hämodialyse-Patienten, Sexarbeiter innen und Menschen, die injektionen Medikamente verwenden, sind einige der wichtigsten Risikogruppen für eine Virushepatitis-Infektion. Darüber hinaus wurde festgestellt, dass von 1999 bis 2018 ein großer Teil der bestätigten Fälle von Hepatitis (21.844 Fälle) Personen mit unvollständiger Schulbildung von der fünften bis zur achten Klasse der Grundschule entsprach.

Hepatitis B und C sind die häufigsten bei virushepatitis. In den letzten Jahren war ein deutlicher Anstieg (mehr als $90 \%$ ) Übertragung von Hepatitis B sexuell. Die Übertragung von Hepatitis $C$ ist eine Freude, und ihr Potenzial für sexuelle Übertragbarkeit gilt als begrenzt und wird daher nicht als STDs betrachtet.

Einer der wichtigsten Faktoren und Faktoren für die Rate der infizierten HepatitisViren ist ihre Koinfektion mit HIV. Etwa $70 \%$ bis $90 \%$ der HIV-positiven Patienten haben Marker einer früheren HBV-Infektion.

Bei Virushepatitis werden klinische und Laborkriterien berücksichtigt. Anzeichen und Symptome wie Gelbsucht, Cholurie, weißlich gefärbte Stühle, Müdigkeit, Unwohlsein, Übelkeit, und andere können bei dem Patienten mit Hepatitis auftreten. Es kann jedoch auch asymptomatische Fälle geben, die zur Verzögerung der Diagnose und der daraus resultierenden Chronik der Krankheit beitragen. Labortests

RC: 67677

Verfügbar in: https://www.nucleodoconhecimento.com.br/gesundheit/virushepatitis 
(Immunoassay, molekulare Tests) sollten durchgeführt werden, um Marker zu erkennen und das ätiologische Mittel zu bestimmen, das die Pathologie verursacht.

\section{REFERENZEN}

ALMEIDA, C. S. C.; MARTINS, L. C. Soroepidemiologia do vírus da hepatite C em cônjuges de portadores desse vírus. Revista Paranaense de Medicina, v. 29, n.1, p. 11-16, 2015.

BRASIL. Ministério da Saúde. Portal da Saúde. PRENVENÇÃO-HEPATITES: HEPATITE D. Disponível em:< http://portalsaude.saude.gov.br/index.php/links-deinteresse/305-hepatites-virais/prevencao-hepatites/9124-hepatite-d> . 2014.

BRASIL. Ministério da Saúde. Portal da Saúde. PRENVENÇÃO-HEPATITES: HEPATITE B. Disponível em:<http://portalsaude.saude.gov.br/index.php/links-deinteresse/305-hepatites-virais/prevencao-hepatites/9130-hepatite-b > . 2014a.

BRASIL, 2015. Ministério da Saúde. Boletim Epidemiológico Hepatites virais. Ano IV, n. 01, 2015.

BRASIL, 2018a. Ministério da Saúde. Boletim Epidemiológico Hepatites Virais. V. 49, 2018.

BRASIL, 2018b. Ministério da saúde. Manual técnico para o diagnóstico das hepatites virais. 2018.

BRASIL, 2019. Ministério da Saúde. Boletim Epidemiológico Hepatites Virais. V.50, 2019.

FARIAS, N.; SOUZA, I.; COELHO, D. M.; OLIVEIRA, U. B.; BINELLI, C. A. Coinfecção pelos vírus das hepatites B ou C e da imunodeficiência adquirida: estudo exploratório no estado de São Paulo, Brasil, 2007 a 2010. Epidemiol. Serv. Saúde, v. 21 , n. 3, p. 475-486, 2012.

$\mathrm{RC}: 67677$

Verfügbar in: https://www.nucleodoconhecimento.com.br/gesundheit/virushepatitis 
FERREIRA, C. T.; SILVEIRA, T R. Hepatites virais: aspectos da epidemiologia e da prevenção. Rev. bras. epidemiol., v. 7, n. 4, p. 473-487, 2004 .

FERREIRA, A.R.; FAGUNDES, E.D.T.; QUEIROZ, T.C.N.; PIMENTA, J.R.; JÚNIOR, R.C. N.. Hepatites Virais A, B e C em crianças e adolescentes. Rev Med de Minas Gerais, (Supl 2): S46-S60, 2014.

FRANCISCO, P.M.S.B; DONALISIO, M.R; GABRIEL, F.J.O; BARROS, M.B.A. Vacinação contra hepatite $B$ em adolescentes residentes em Campinas, São Paulo, Brasil. REV BRAS EPIDEMIOL, 18(3): 552-567, JUL-SET 2015.

GOMES, R.; NASCIMENTO, E. F.; ARAÚJO, F. C. Por que os homens buscam menos os serviços de saúde do que as mulheres? As explicações de homens com baixa escolaridade e homens com ensino superior. Cad. Saúde Pública, v. 23, n.3, p. $565-574,2007$.

GUSMÃO, K.E et al. PERFIL CLÍNICO-EPIDEMIOLÓGICO DA HEPATITE C NA REGIÃO NORTE DO BRASIL ENTRE 2012 E 2015.Revistade Patologia do Tocantins, [S.I.], v. 4, n. 2, p. 41-45, jun. 2017.

LEITE, A.C.D. et al. . Avaliação das Internações Pela Hepatite Aguda B em Comparativo com a Aplicação de Doses da Vacina Contra Hbv, Na Região Norte. In: Luís Marcelo Aranha Camargo; Dionatas Ulises de Oliveira Meneguetti; Jader de Oliveira. (Org.). Atualidades em Medicina Tropical no Brasil: Educação em Saúde. 1ed.Rio Branco, Acre: Stricto Sensu Editora, 2020, p. 39-48.

MARGREITER, S. et al. Estudo de prevalêcia das hepatites virais b e c no município de palhoça-sc. Rev. Saíde Públ. Santa Cat., Florianópolis, v. 8, n. 2, p. 21-32, maio/ago. 2015.

MOCBEL, I.L.S.A. et al. CONHECIMENTO SOBRE HEPATITES A e E DOS ESTUDANTES DE UMA ESCOLA MUNICIPAL EM SANTARÉM - PA. Revista EM FOCO - Fundação Esperança/IESPES, [S.I.], v. 2, n. 24, p. 18-29, abr. 2016.

RC: 67677

Verfügbar in: https://www.nucleodoconhecimento.com.br/gesundheit/virushepatitis 
NUNES, H.M. et al. Soroprevalência da infecção pelos vírus das hepatites A B, C, D e E em município da região oeste do Estado do Pará, Brasil. Rev. Pan-Amaz. Saúde, Ananindeua, v. 7, n. 1, p. 55-62, mar. 2016.

SBI (SOCIEDADE BRASILEIRA DE INFECTOLOGIA). Boletim terapêutico de HIV/Aids, DTS e Hepatites Virais. Ano I, n. 4, 2003.

SILVA, C.M; VIANNA, G.S.P; SOARES, M.C.P; AMARAL, I.S.A.; MOIA, L.J.M.P. AVALIAÇÃO DO HEMOGRAMA EM PACIENTES TRATADOS PARA HEPATITE C1. Revista Paraense de Medicina - V.28 (2) abril-junho 2014.

Eingereicht: November 2020.

Genehmigt: November 2020. 